\title{
Let's Grow Together: Tutorials on Learning Analytics Methods
}

\author{
Dragan Gašević \\ The University of Edinburgh, United Kingdom \\ dgasevic@acm.org \\ Mykola Pechenizkiy \\ Eindhoven University of Technology, The Netherlands
}

\begin{abstract}
This paper is a guest editorial into a special section that offers a collection of tutorials on methods that can be used in learning analytics. The special section is prepared as a response to the growing need of learning analytics practitioners and researchers to learn and use novel methods. In spite of this need, papers that systematically introduce some of the methods have been underrepresented in the literature. Specifically, the special section features papers that introduce epistemic network analysis, automated content and network analysis of social media, text coherence analysis with Coh-Metrix, microgenetic analysis with sequence pattern mining, and design of visual learning analytics guided by educational theory informed goals.
\end{abstract}

Keywords: learning analytics, tutorials, text analysis, social network analysis, epistemic network analysis, microgenetic learning, sequence pattern mining, information visualization

\section{INTRODUCTION}

The field of learning analytics has frequently been characterized as bricolage (Dawson, Gašević, Siemens, \& Joksimovic, 2014) by drawing on the body of existing knowledge from any related disciplines such as educational psychology, learning sciences, machine learning, text mining, and human computer interaction. This mix of different fields creates a productive "middle space" (Suthers \& Verbert, 2013) that offers many promising opportunities for advancing our understanding and optimization of learning and environments in which learning happens. Frequently, in learning analytics, we can found papers that look at the ways to explain factors that shape learning outcomes and processes. Different methods from statistics and data mining are commonly used to address some of these goals. To get deeper insights into different learning processes, text analysis of learning discourse and social network analysis are frequently suggested. Insights obtained through some of these analyses are eventually to be presented to learners and educators to offer feedback with an aim to provide educators and learners with insights they can act on to improve learning processes and teaching practice. More recently, several authors stressed a need to build and interpret statistical models and feedback tools by building on established theories of learning and education research (Gašević, Dawson, \& Siemens, 2015; Wise \& Shaffer, 2015). 
(2016). Let's grow together: Tutorials on learning analytics methods. Journal of Learning Analytics, 3(3), 5-8. http://dx.doi.org/10.18608/jla.2016.33.2

Although highly promising, this mix of fields also requires from researchers and practitioners to develop some understanding of other disciplines to work effectively in interdisciplinary teams and to unlock the full potential of learning analytics. However, researchers and practitioners come with different epistemic perspectives and frequently use different research and analytical methods. Therefore, for a healthy development of the field of learning analytics, opportunities for researchers to develop both their conceptual understanding and their skills in different methods are necessary. The creation of such opportunities is not an easy task and requires mobilization of a wide range of individuals and organizations who can cater to diverse needs, perspectives, and backgrounds of those interested in advancing their learning analytics skills. The learning analytics community recognized this need early and started organizing opportunities for skill development at different learning analytics events. The two main annual events (Learning Analytics Summer Institute, LASI and International Conference on Learning Analytics \& Knowledge, LAK) organized by the Society for Learning Analytics Research (SoLAR), often in partnership with the sister International Educational Data Mining Society (IEDMS), have offered a wide range of tutorials and workshops that specifically introduce different analytic methods and approaches (e.g., machine learning for education researchers). These workshops and tutorials received much interest and appreciation by the members of the community. Yet to date, papers that systematically introduce some of the methods presented at the workshops and tutorials that can be used by others, have not been provided.

\section{PAPERS IN THIS SECTION}

This special section brings five papers that were prepared based on the tutorials and workshops organized at LAK in Indianapolis, IN and LASI at Harvard University in 2014. In the first paper of this collection, Shaffer, Collier, and Ruis present a tutorial on epistemic network analysis (ENA). ENA is a method well established in the learning sciences (Shaffer et al., 2009) and is commonly used for analysis of the results of a content analysis in which learning processes (e.g., emerging in learning discourse) are coded by using different coding schemes. ENA has a strong statistical foundation that allows for analysis of networks built based on co-occurrence of codes in artifacts of interests (e.g., in the same message posted to an online discussion board). The tutorial presented by Shaffer and his colleagues provides an introduction into the theoretical foundations behind ENA practical steps necessary to prepare and analyze data in ENA. This is followed by the introduction of the ENA tools, analysis methods available in the tools, and most importantly the ways how results of ENA can be interpreted.

A tutorial on the analysis of learning in social media with content and social network analysis is prepared by Gruzd, Paulin, and Haythornthwaite. The tutorial gives an overview of the ways how social networks can be extracted from different social media and processed with different metrics commonly used in social network analysis. Gruzd and his colleagues also recognize that, while social networks can offer useful insights in terms of the flow of information and social structures, they typically do not offer 
(2016). Let's grow together: Tutorials on learning analytics methods. Journal of Learning Analytics, 3(3), 5-8. http://dx.doi.org/10.18608/jla.2016.33.2

sufficient insight into the content that has been communicated via social networks. To address this need, Gruzd and his colleagues look into content analysis by using tools for automated text analysis.

The introduction of methods for text analysis is further expanded by Dowell, Graesser, and Cai (this issue) who offer a tutorial into Coh-Metrix (Graesser, McNamara, \& Kulikowich, 2011) . Coh-Metrix is framework for coherence analysis of learning discourse and is rooted into research on text comprehension. The tutorial first provides a theoretical foundation behind Coh-Metrix, which is followed by the description of metrics and principle components commonly used as part of Coh-Metrix. The tutorial then offers a brief description of the web-based Coh-Metrix tool and the ways how CohMerix can be used to address a wide range of topics in educational research.

Analysis of temporal dimensions of learning receives more attention in learning analytics due to the developmental nature of learning, typically characterized by sequences of operations and processes performed by learners (Winne, 2014). Aghababyan, Martin, Janisiewicz, and Close provide a tutorial into microgenetic methods that can be used to uncover temporal patterns of learning from digital traces. Aghababyan and her colleagues specifically illustrate how $\mathrm{R}$, a popular statistical language, can be used for uncovering sequence patterns of learning on a data set generated by 52 elementary school students by using a digital learning environment.

The special section is concluded by the paper by Hillaire, Schlichtmann, and Ducharme with a tutorial on a method used for prototyping visual learning analytics. Although information visualization is frequently used in learning analytics (Verbert, Duval, Klerkx, Govaerts, \& Santos, 2013), there has been much less literature that offers methodological approaches how such learning analytics visualizations should be designed. More importantly, how such visualizations can be designed by being guided by educational theory informed goal. The paper provides a design method that fills this gap in the literature and illustrates the use of the method on a case study of the creation of a self-reported emotional measure.

We hope that you will find the papers in this special section useful for your future research, practice, and teaching in learning analytics. We would also like to encourage you to prepare similar papers on other relevant methods for learning analytics and submit them to the journal, to teach others about the approach, and support the development of the field of learning analytics as a whole.

\section{REFERENCES}

Dawson, S., Gašević, D., Siemens, G., \& Joksimovic, S. (2014). Current state and future trends: A citation network analysis of the learning analytics field. Proceedings of the Fourth International Conference on Learning Analytics and Knowledge (LAK'14), 231-240. https://dx.doi.org/10.1145/2567574.2567585

Gašević, D., Dawson, S., \& Siemens, G. (2015). Let's not forget: Learning analytics are about learning. TechTrends, 59(1), 64-71. https://doi.org/10.1007/s11528-014-0822-x 
(2016). Let's grow together: Tutorials on learning analytics methods. Journal of Learning Analytics, 3(3), 5-8. http://dx.doi.org/10.18608/jla.2016.33.2

Graesser, A. C., McNamara, D. S., \& Kulikowich, J. M. (2011). Coh-Metrix Providing Multilevel Analyses of Text Characteristics. Educational Researcher, 40(5), 223-234. https://dx.doi.org/10.3102/0013189X11413260

Shaffer, D. W., Hatfield, D., Svarovsky, G. N., Nash, P., Nulty, A., Bagley, E., ... Mislevy, R. (2009). Epistemic network analysis: A prototype for 21st-century assessment of learning. International Journal of Learning and Media, 1(2), 33-53. https://dx.doi.org/10.1162/ijlm.2009.0013

Suthers, D., \& Verbert, K. (2013). Learning analytics as a "middle space." Proceedings of the Third International Conference on Learning Analytics and Knowledge, 1-4. https://dx.doi.org/10.1145/2460296.2460298

Verbert, K., Duval, E., Klerkx, J., Govaerts, S., \& Santos, J. L. (2013). Learning Analytics Dashboard Applications. American Behavioral Scientist, 57(10), 1500-1509. https://dx.doi.org/10.1177/0002764213479363

Winne, P. H. (2014). Issues in researching self-regulated learning as patterns of events. Metacognition and Learning, 1-9. https://dx.doi.org/10.1007/s11409-014-9113-3

Wise, A. F., \& Shaffer, D. W. (2015). Why theory matters more than ever in the age of big data. Journal of Learning Analytics, 2(2), 5-13. https://dx.doi.org/10.18608/jla.2015.22.2 